\title{
USO DE COLÓIDES E CRISTALÓIDES NO PERIOPERATÓRIO DE PACIENTES GRAVES: CONTROVERSIAS
}

\section{ARTIGO DE REVISÃO}

TAVARES, Marcela Marçolla ${ }^{1}$

TAVARES, Marcela Marçolla. Uso de Colóides e Cristalóides no perioperatório de pacientes graves: Controversias. Revista Científica Multidisciplinar Núcleo do Conhecimento. Ano 05, Ed. 01, Vol. 01, pp. 05-21. Janeiro de 2020. ISSN: 2448-0959, Link de acesso: https://www.nucleodoconhecimento.com.br/saude/coloides-ecristaloides

\section{RESUMO}

Como o manejo da reposição de fluidos influência o prognóstico do paciente, a pesquisa básica e clínica vem abordando os vários aspectos diferentes que contribuem para a administração de líquidos e volume no período perioperatório. Ainda não existe um consenso sobre quando e em quem indicar a otimização hemodinâmica perioperatória. Questões adicionais como o papel relativo da otimização da relação cristalóide versus colóide não foram resolvidas. Este trabalho constitui-se de uma revisão da literatura, realizada entre fevereiro e outubro de 2019, no qual realizou-se uma consulta a publicações científicas variadas, presentes na internet, por meio de consulta nas bases de dados: ScienceDirect, UptoDate, Medline/PubMed, Lilacs, sciELO, com os termos Fluidoterapia Perioperatória, Hemodinâmica, Reposição Volêmica, Cristalóide, Coloide. A partir disso, observou-se que, os fluidos devem ser aplicados como qualquer outro medicamento, respeitando suas indicações e limitações individuais, sendo esta a base do conceito da Terapia Guiada por Objetivos - novo conceito que encoraja o enquadramento do paciente em critérios de inclusão para a otimização fluída. Referindo-se a isso, aspectos vantajosos da reposição volêmica perioperatória com soluções iso-oncóticas são

\footnotetext{
${ }^{1}$ Médica. Pós graduada em Medicina Intensiva para Adultos.
} 
compreensíveis, embora sejam necessários ensaios clínicos randomizados controlados adicionais que provem sua relevância para o desfecho.

Palavras-chave: Fluidoterapia Perioperatória, Hemodinâmica, Reposição Volêmica.

\section{INTRODUÇÃO}

Como o manejo da reposição de fluidos influencia o prognóstico do paciente, a pesquisa básica e clínica vem abordando os vários aspectos diferentes que contribuem para a administração de líquidos e volume no período perioperatório. A pesquisa básica melhorou o conhecimento sobre a função da barreira vascular endotelial e suas alterações funcionais responsáveis pelo extravasamento vascular. Estudos clínicos, proclamando diferentes abordagens para o controle de fluidos, mostraram resultados conflitantes e, na maioria dos casos, não se referem à base fisiológica da barreira vascular. Com base na meta-análise dos últimos 30 anos, tem havido uma série de estudos realizados para reduzir a mortalidade e a morbidade cirúrgica por manipulação deliberada e preventiva da hemodinâmica perioperatória, evidenciando uma tendência da comunidade científica em esclarecer se a optimização hemodinâmica é de fato benéfica[2].

O mesmo responde por estudos que se concentraram principalmente nos objetivos clínicos para orientar a terapia volumétrica no perioperatório. No entanto, um raciocínio deve geralmente ser derivado de fatos fisiológicos e estudos significativos e comparáveis. Assim, esta revisão resume o conhecimento relevante sobre o efeito de diferentes fluidos intravenosos e sobre o monitoramento hemodinâmico. Como ainda não há um consenso nesse assunto de grande interesse a todos os anestesiologistas, o objetivo deste artigo é esclarecer algumas vantagens e desvantagens dos principais fluidos utilizados na reposição volêmica perioperatória.

O papel relativo da otimização da relação cristalóide versus colóide, apesar de não ter sido resolvido, há evidências que favorecem soluções equilibradas com uma concentração de eletrólito semelhante à encontrada no plasma e, grandes volumes de solução salina devem ser evitada, pois induzem acidose hiperclorêmica[3]. 


\section{MATERIAL E MÉTODOS}

Este trabalho constitui-se de uma revisão da literatura, realizada entre fevereiro e outubro de 2019, no qual realizou-se uma consulta a publicações científicas variadas, presentes na internet, através de busca, a partir das bases de dados de ScienceDirect, UptoDate, Medline/PubMed, Lilacs, sciELO, principalmente com os termos Fluidoterapia Perioperatória, Hemodinâmica, Reposição Volêmica, Cristalóide, Coloide.

Os critérios para inclusão neste trabalho foram artigos científicos que descrevem as principais diferenças entre coloides e cristaloides bem como suas principais indicações no período perioperatório. Diversas modalidades de estudos entre os anos de 1980 e 2019 foram revistas, incluindo estudos prospectivos randomizados e multicêntricos, meta-análises, estudos observacionais retrospectivos e revisões de literatura.

\section{REVISÃO}

\subsection{A FISIOLOGIA DA COMPARTIMANTALIZAÇÃO DE FLUIDOS}

A água corporal total para um indivíduo de $75 \mathrm{~kg}$ é de aproximadamente $45 \mathrm{~L}(60 \%)$. Dois terços disso $(30 \mathrm{~L})$ são água intracelular. $\mathrm{O}$ terço remanescente $(15 \mathrm{~L})$ no compartimento extracelular é dividido entre os compartimentos intravascular $(3 \mathrm{~L})$ e extravascular (12 L) (Fig. 1)[4]. O volume intravascular total é de aproximadamente 5 $\mathrm{L}$ e tem componentes celulares (glóbulos vermelhos e brancos e plaquetas: $40 \%$ [2 L]) e não celulares (plasma: 60\% [3 L]). O plasma é uma solução aquosa de íons inorgânicos (predominantemente cloreto de sódio), moléculas simples, como a uréia, e moléculas orgânicas maiores, como a albumina e as globulinas. 
FIGURA 01: Modelo explicativo da distribuição de fluidos no organismo

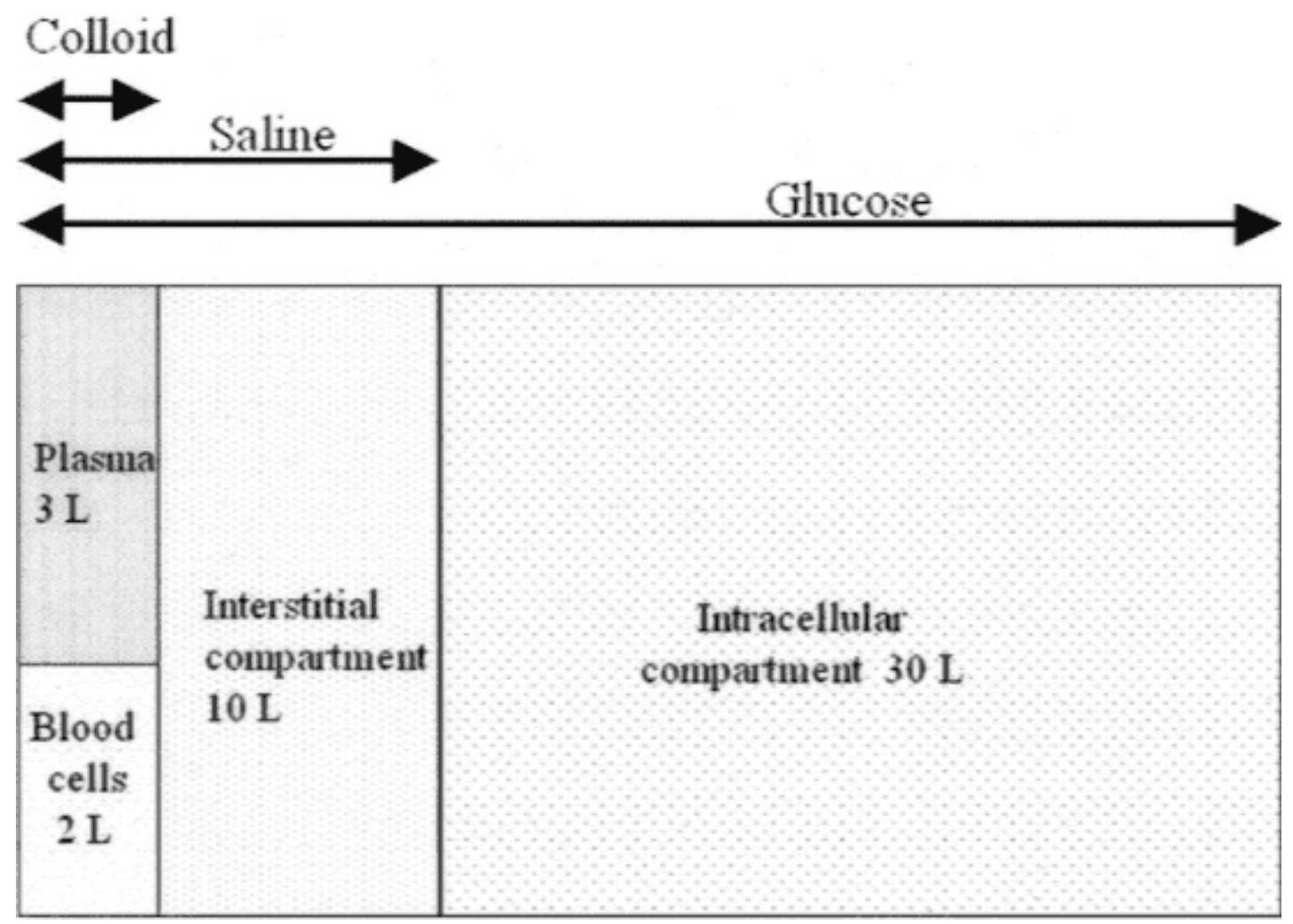

Fonte: Grocott et al. (2005)

Conforme representado na Figura 01, nota-se que grande parte do volume líquido se concentra no meio intracelular, sendo composto por solução glicosada.

O aumento da permeabilidade vascular devido à ativação inflamatória com comprometimento da função celular endotelial pode ocorrer por uma variedade de razões durante procedimentos cirúrgicos maiores[5]. Especificamente, trauma de tecido cirúrgico, hipoperfusão tecidual decorrente de fluidoterapia inadequada, lesão de isquemia / reperfusão, sepse (local ou sanguínea) e uso de circulações extracorpóreas (por exemplo, circuito de circulação extracorpórea) são reconhecidos como estímulos inflamatórios que podem comprometer a função vascular[6]. 


\subsection{REPOSIÇÃO VOLÊMICA}

A reposição volêmica deveria estar indicada todas as vezes que houver necessidade de: 1) substituir perdas sanguíneas durante a cirurgia, 2) Substituir perdas por transpiração e produção de urina 3) Pré-operatório de cirurgias do intestino e 4) manutenção das perdas do terceiro espaço[7].

Entenda-se por terceiro espaço a como sendo um termo proposto por Randall em 1952, para descrever a situação na qual o líquido extracelular é perdido ou sequestrado numa área do corpo onde não participa das trocas, e consequentemente não satisfaz às necessidades hídricas do paciente[8].

\subsection{ESTRATÉGIA DE OTIMIZAÇÃO HEMODINAMIDA PERIOPERATÓRIA}

Os pacientes que poderiam se beneficiar desta terapêutica são aqueles com elevado risco segundo o índice de Risco Cardíaco Revisado e o teste de Exercício Cardiopulmonar Pré-operatório[6],[9],[10]

Conforme revisão sistemática de literatura acerca da otimização Peri operatória no paciente com alto risco cirúrgico, constatou-se importantes fatores que ajudariam a identificar os pacientes que mais se beneficiariam da otimização de fluidos perioparatória[11]. Os resultados são descritos na Tabela 01, com base nos estudos de Mangano et al. (1996), Poldermans et al. (1999) e Shoemaker et al. (1988).

TABELA 01 - Critérios de inclusão para a otimização perioperatória

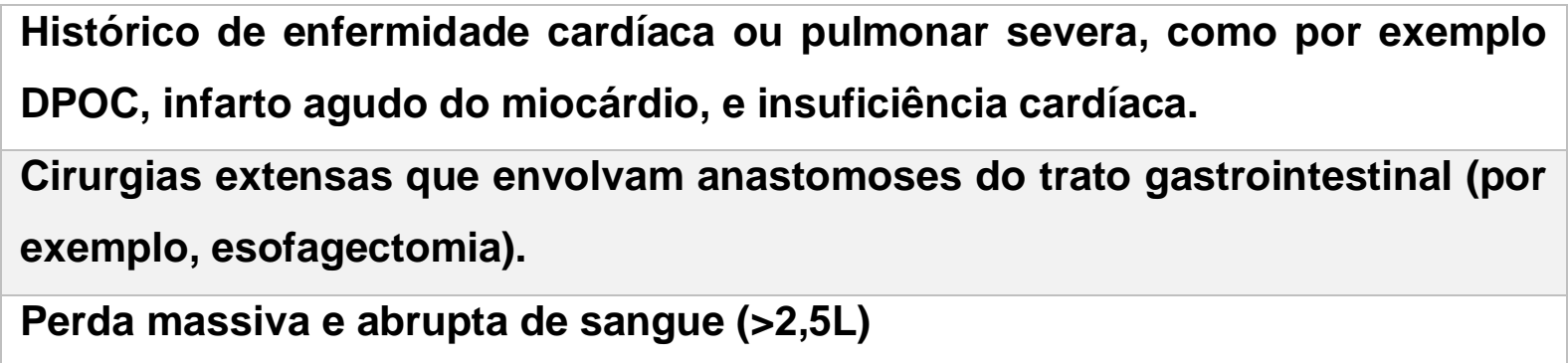


Idade maior ou igual a 70 anos com reserve fisiológica limitada em um ou mais órgãos vitais.

\section{Septicemia}

Insuficiência Respiratória (PaO2 <60 mmHg e FIO2 >0.4)

Acometimento abdominal agudo como pancreatite, perfuração de vísceras ou sangramentos do TGI.

\section{Insuficiência Renal Aguda}

\section{Doença vascular em estágio avançado}

Fonte: Tote; Grounds (2006)

Com base em estudo envolvendo 30 pacientes graves, que sofreram cirurgias cardíacas, evidenciou-se que apesar do aumento do índice cardíaco e do fluxo sanguíneo renal com ambos os fluidos, nenhum dos fluidos melhorou a oxigenação renal, porque ambos induziram hemodiluição. A TFG aumentou no cristaloide (28\%), mas não no grupo colóide, conforme apresentado na Figura 02[12].

O mesmo estudo mostrou que o uso de cristalóides aumentou a taxa de filtração (24\%) bem como a extração renal de oxigênio (23\%), mesmo sem aumento da oferta vascular do componente, sugerindo que o aumento na TFG - o principal determinante do consumo de oxigênio - não foi acompanhado por um aumento proporcional na fração de oxigênio fornecido aos rins. Em outras palavras, seus resultados resumem em gráfico (figura 02) que o cristalóide aumentou a extração renal de oxigênio ( $P$ $<0,001$ ) 20, 40 e 60 min após o bolus, em contraste com o colóide (NS), sugerindo comprometimento da oxigenação renal no segundo caso. A mudança na extração renal de oxigênio foi significativamente $(P<0,05)$ mais pronunciada no grupo cristaloide em comparação com o grupo colóide[11]. 
FIGURA 02: Efeitos da infusão de cristaloide $(10 \mathrm{ml} / \mathrm{kg})$ e de coloide $(20 \mathrm{ml} / \mathrm{kg}) \mathrm{na}$ extração renal de oxigênio após cirurgia cardíaca

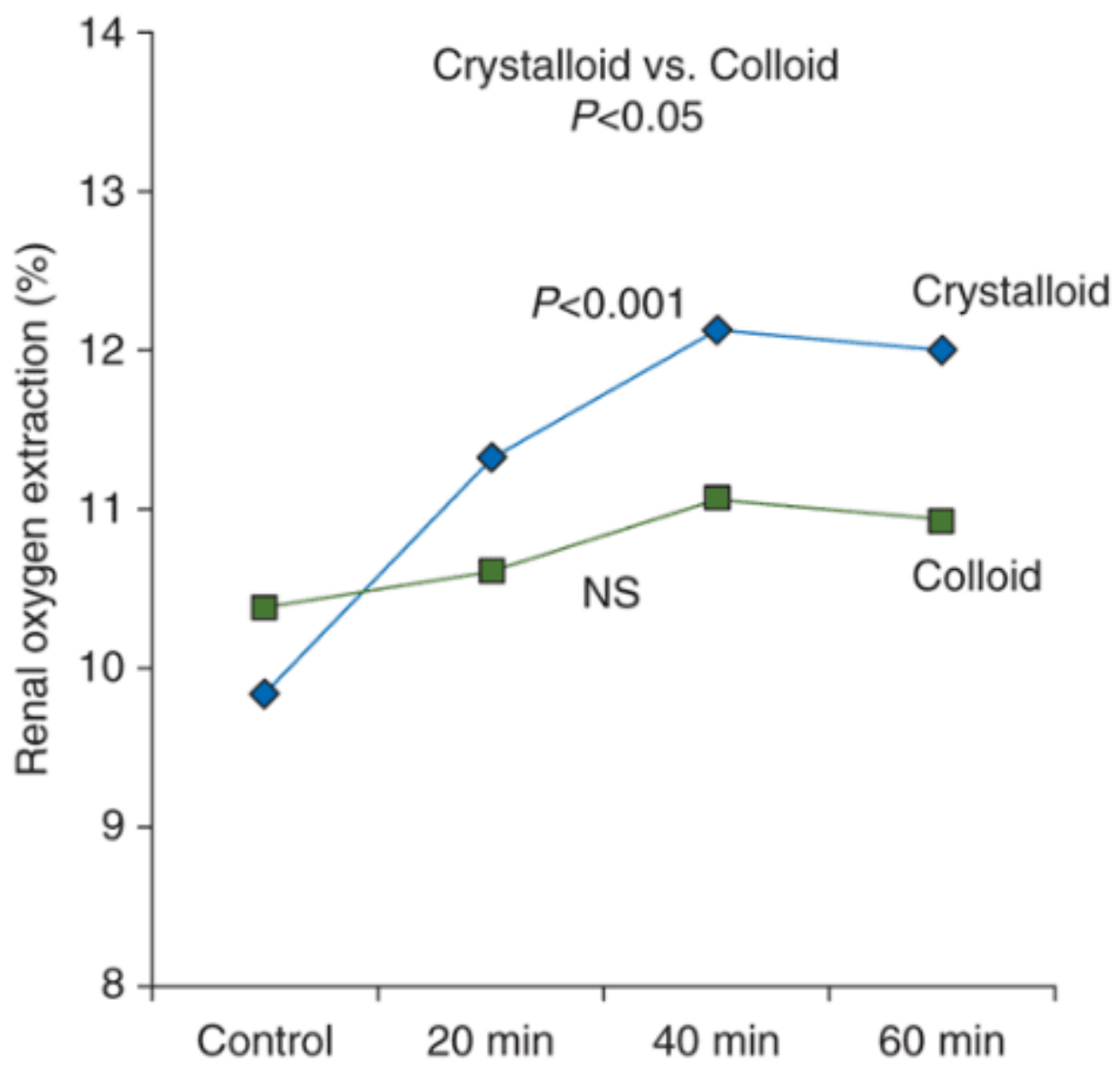

Fonte: Skytte Larsson. et al. (2015)

\subsection{O TIPO DE FLUIDO PARA A ESTRATÉGIA DE OTIMIZAÇÃO HEMODINÂMICA}

Inúmeras apresentações de soluções estão disponíveis para a reposição volêmica, elas variam de acordo com a composição de íons e pela presença ou ausência de compostos orgânicos. Assim temos a presença de coloides e cristaloides.

Coloides são considerados expansores plasmáticos por provocarem menos perda capilar e menos edema pulmonar que os cristaloides. Eles reduzem a expressão de mediadores inflamatórios, melhoram a microcirculação e oxigenação tecidual e 
promovem ressuscitação volêmica superior à dos cristaloides. Entretanto, efeitos colaterais (EC) já foram observados[5].

Os cristaloides, em contrapartida, deixam o compartimento intravascular mais cedo e em maior quantidade do que os colóides e, portanto, um volume maior é necessário para repor déficits (3 a 4 vezes o volume de cristalóides)[6]. Algumas das principais características dos cristaloides são apresentadas nas tabelas 02 e dos colóides na tabela 03[3].

TABELA 2 - Principais cristalóides e suas respctivas Osmolaridades e concentrações de Sódio $(\mathrm{Na}+)$ e Potássio $(\mathrm{K}+)$

\begin{tabular}{|l|l|l|l|}
\hline Cristaloide & Osmolaridade (mOsm/L) & $\mathrm{Na+}$ & $\mathrm{K}+$ \\
\hline Glucose 5\% & 252 & - & - \\
\hline $\begin{array}{l}\text { Glucose 25\% } \\
\text { Glucose 50\% }\end{array}$ & 1260 & - & - \\
\hline $\begin{array}{l}\text { Sodium chloride } \\
\text { 0.9\% }\end{array}$ & 308 & - & 154.0 \\
\hline $\begin{array}{l}\text { Sodium chloride } \\
\text { and glucose }\end{array}$ & 264 & 154.0 & 31.0 \\
\hline $\begin{array}{l}\text { Ringer's solution } \\
\text { Compound sodium }\end{array}$ & 278 & 31.0 & \\
\hline $\begin{array}{l}\text { lactatea } \\
\text { Plasmalyte B }\end{array}$ & 298.5 & 147.0 & 156.0 \\
\hline Normasol & 280 & 131.0 & 111.0 \\
\hline
\end{tabular}

Fonte: Adaptado de Grocott. Et al (2005)

Em um estudo prospectivo, cego, em pacientes eletivos que cirurgia não-cardíaca, ressuscitação fluida intra-operatória com uso de colóide $(6 \% \mathrm{HES})$ reduziu-se a incidência de náuseas e vômitos no pós-operatório, dor intensa, edema periorbital e visão dupla[13]. 
Em pacientes submetidos a cirurgia abdominal de grande porte, quando receberam cristaloides sozinhos resultaram em acúmulo de líquido intestinal comparado com aqueles que também recebeu colóides (6\% HES)[14]. Isso leva ao aumento do edema tecidual. A associação de edema tecidual com comprometimento na perfusão e oxigenação é controversa[15]

TABELA 3: Principais coloides e seus respectivos valores de Peso Molecular Médio Ponderado(PMMP), Numero Molecular Médio Ponderado (NMMP) e concentrações de Sódio $(\mathrm{Na}+)$ e Cloro (Cl-)

\begin{tabular}{|l|l|l|l|l|l|}
\hline Solução & Coloide & PMMP(Da) & $\begin{array}{l}\text { NMMP } \\
(\mathrm{Da})\end{array}$ & $\mathrm{Na}+(\mathrm{mmol} / \mathrm{L})$ & $\begin{array}{l}\mathrm{Cl}- \\
(\mathrm{mmol} / \mathrm{L})\end{array}$ \\
\hline $\begin{array}{l}\text { Gelofusine } \\
\mathbf{( 4 \% )}\end{array}$ & $\begin{array}{l}\text { Succinylated } \\
\text { gelatin }\end{array}$ & 30,000 & 22,600 & 154 & 125 \\
\hline $\begin{array}{l}\text { Haemaccel } \\
\mathbf{( 3 . 5 \% )}\end{array}$ & Polygeline & 35,000 & 24,300 & 145 & 145 \\
\hline Voluven & Tetrastarch & 130,000 & 60,000 & 154 & 154 \\
\hline Pentaspan & Pentastarch & 264,000 & 63,000 & 154 & 154 \\
\hline HAES-steril 6\% & Pentastarch & 200,000 & 60,000 & 154 & 154 \\
\hline or 10\% & & & & & \\
\hline EloHase 6\% & Hexastarch & 200,000 & 60,000 & 154 & 154 \\
\hline Hespan 6\% & Hetastarch & 450,000 & 70,000 & 150 & 150 \\
\hline Hextend & Hetastarch & 670,000 & 70,000 & 143 & 124 \\
\hline Gentran 40 & Dextran 40 & 40,000 & 25,000 & 154 & 154 \\
\hline Gentran 70 & Dextran 70 & 70,000 & 39,000 & 154 & 154 \\
\hline Rheomacrodex & Dextran 40 & 40,000 & 25,000 & 154 & 154 \\
\hline Macrodex & Dextran 70 & 70,000 & 39,000 & 154 & 154 \\
\hline
\end{tabular}

Adaptado de Grocott; Mythen; GAN (2005)

Por outro lado, grandes quantidades de solução salina levam a acidose hiperclorêmica[16] e ao estado de hipercoagulobilidade[17] ainda mais evidenciado do que o normalmente visto na infusão de outros cristaloides[18]. 


\subsection{USO RACIONAL DE FLUIDOS NO PERIOPERATÓRIO}

Mais do que o tipo de fluido utilizado, há uma preocupação cada vez mais clara em identificar às reais indicações da reposição de fluidos no perioperatório.

Uma das estratégias que mais prevalece é a terapia dirigida por Objetivos (TGO), que tem sido repetidamente usada para melhorar significativamente os resultados da otimização hemodinâmica, tanto a curto como a longo prazo[19][[20] A abordagem TGO é centrada na maximização (otimização) do débito cardíaco (CO) pela administração incremental de fluidos[21].

O consenso Nacional realizado em 2016 concluiu que o sucesso na Terapia Guiada por Objetivos (TGO) quanto outras estratégias restritivas de fluidos sugerem que, o planejamento de fluidos no perioperatório deve enfatizar que a fluidoterapia seja administrada somente quando há uma indicação clara[22] utilizando para tal, os critérios de inclusão apresentados na tabela 01.

Ainda, quanto a estratégia terapêutica direcionada, há 9 estudos em que uma estratégia terapêutica direcionada a um objetivo também foi utilizada para maximizar variáveis hemodinâmicas relacionadas ao fluxo pacientes cirúrgicos, intra e pósoperatório[23]. Os estudos constataram que a estratégia de tratamento reduziu as complicações gastrointestinais e tempo de internação hospitalar[24], tanto na enfermaria como nas unidades de terapia intensiva[25].

De maneira concomitante ao início da terapia é importante ressaltar que, uma vez iniciada, a avaliação da responsividade antes da administração de fluidos pode não apenas ajudar na detecção de pacientes com necessidade de líquidos, mas também evitar sobrecarga desnecessária e prejudicial de fluidos. Esta avaliação consiste, por exemplo, em observar os parâmetros dinâmicos funcionais como alterações no débito cardíaco durante manobras modificadoras da pré-carga - como a elevação passiva dos membros inferiores[20]. 
Em pacientes cirúrgicos de alto risco, o uso de um protocolo hemodinâmico para manter a perfusão tecidual diminuiu a mortalidade e a falência de órgãos no pósoperatório[26]. Monitorar o débito cardíaco calculando o transporte e o consumo de oxigênio também ajudou a orientar a terapia[18].

\section{CONSIDERAÇÕES FINAIS}

O esquema de reposição ideal seria aquele que diminuísse a mortalidade e melhorasse a qualidade de vida, diminuísse a insuficiência de múltiplos órgãos e o uso de sangue e tivesse baixo custo. Este esquema obviamente ainda não foi alcançado. Como não há diretriz baseada em evidência, na prática utiliza-se combinação de cristaloides e coloides, sendo mais importante saber como repor do que saber o que repor.

Geralmente, os fluidos devem ser aplicados como qualquer outro medicamento, respeitando suas indicações e limitações individuais. Referindo-se a isso, aspectos vantajosos da reposição volêmica perioperatória com soluções iso-oncóticas são compreensíveis, embora sejam necessários ensaios clínicos randomizados controlados adicionais que provem sua relevância para o desfecho. Resumindo os argumentos citados acima, uma estratégia racional de administração de fluidos poderia ser tratar pacientes submetidos à cirurgia de baixo risco com perda insignificante de volume intravascular com infusão de cristaloides e usar uma combinação de administração cristalóide e colóide, cuidadosamente titulada com base em medidas hemodinâmicas.

Concluímos, por fim, que a Terapia Guiada por Objetivos no perioperatório está associada a reduções nas complicações e na duração da internação hospitalar. Os efeitos benéficos da TGO podem ser alcançados, evitando assim as dificuldades da admissão na Unidades de Terapia Intensiva. 


\section{REFERÊNCIAS}

BRANDSTRUP, B.; SVENDSEN, P. E.; RASMUSSEN, M.; et al. Which goal for fluid therapy during colorectal surgery is followed by the best outcome: near-maximal stroke volume or zero fluid balance? British Journal of Anaesthesia. United Kingdom, v. 109, n.2 , p. 191-199, 17 jun. 2012. Disponível em: <https://ac-elscdn.ez3.periodicos.capes.gov.br/S000709121732888X/1-s2.0-S000709121732888Xmain.pdf?_tid=bde7ae63-beb3-46c9-9adce0f04482d0cf\&acdnat=1539636094_9a27cfa1829cc5679acd68973a0e7357>. Acesso em: 15 out. 2019.

BUNDGAARD-NIELSEN, M.; HOLTE, K.; SECHER, N. H.; KEHLET, H. Monitoring of peri-operative fluid administration by individualized goal-directed therapy. Acta Anaesthesiologica Scandinavica. USA v. 51, n. 3, p. 331-340, mar. 2007. Wiley/Blackwell (10.1111). Disponível em: <http://doi.wiley.com/10.1111/j.13996576.2006.01221.x>. Acesso em: 16 out. 2019.

GOW, K. W.; PHANG, P. T.; TEBBUTT-SPEIRS, S. M.; et al. Effect of crystalloid administration on oxygen extraction in endotoxemic pigs. Journal of Applied Physiology. USA, v. 85, n. 5, p. 1667-1675, nov. 1998. American Physiological SocietyBethesda, $\mathrm{MD}$ Disponível em: <http://www.physiology.org/doi/10.1152/jappl.1998.85.5.1667>. Acesso em: 15 out. 2019.

GROCOTT, M. P. W.; MYTHEN, M. G.; GAN, T. J. Perioperative Fluid Management and Clinical Outcomes in Adults. Anesthesia \& Analgesia. USA, v. 100, n. 4, p. 10931106, 2005. Disponível em: <https://insights.ovid.com/crossref?an=00000539200504000-00032>. Acesso em: 15 out. 2019.

GURGEL, S. T.; DO NASCIMENTO, P. Maintaining Tissue Perfusion in High-Risk Surgical Patients. Anesthesia \& Analgesia. USA, v. 112, n. 6, p. 1384-1391, 2011. Disponível em: <http://www.ncbi.nlm.nih.gov/pubmed/21156979>. Acesso em: 16 out. 2019.. 
HAMILTON, M. A.; CECCONI, M.; RHODES, A. A Systematic Review and MetaAnalysis on the Use of Preemptive Hemodynamic Intervention to Improve Postoperative Outcomes in Moderate and High-Risk Surgical Patients. Anesthesia \& Analgesia. USA, v. 112, n. 6, p. 1392-1402, 2011. Disponível em: <http://www.ncbi.nlm.nih.gov/pubmed/20966436>. Acesso em: 16 out. 2019.

HOLTE, K.; SHARROCK, N. E.; KEHLET, H. Pathophysiology and clinical implications of perioperative fluid excess. British Journal of Anaesthesia. United Kingdom, v. 84, n. 4, p. $\quad 622-632, \quad$ oct. 2002.2 Disponível em: <https://www.ncbi.nlm.nih.gov/pubmed/12393365>. Acesso em: 16 out. 2019.

KELLUM, J. A. Saline-induced hyperchloremic metabolic acidosis. Critical care medicine. New York, v. 30, n. 1, p. 259-61, 2002. Disponível em: <http://www.ncbi.nlm.nih.gov/pubmed/11902280>. Acesso em: 15 out. 2019.

LEE, T. H.; MARCANTONIO, E. R.; MANGIONE, C. M.; et al. Derivation and Prospective Validation of a Simple Index for Prediction of Cardiac Risk of Major Noncardiac Surgery. Circulation. Dallas, v. 100, n. 10, sep. 1999. Disponível em: < https://www.ncbi.nlm.nih.gov/pubmed/10477528>. Acesso em: 15 out. 2019.

LORENTZ, M. N. Reposição volêmica perioperatória. Rev Med Minas Gerais. Minas Gerais, v. 20, n. 4, p. 47-56, 2018. Disponível em: <http://rmmg.org/artigo/detalhes/1025>. Acesso em: 15 out. 2019.

MANGANO, D. T.; LAYUG, E. L.; WALLACE, A.; TATEO, I. Effect of Atenolol on Mortality and Cardiovascular Morbidity after Noncardiac Surgery. New England Journal of Medicine. Canada, v. 335, n. 23, p. 1713-1721, 1996. Massachusetts Medical Society Disponível

em: <http://www.nejm.org/doi/abs/10.1056/NEJM199612053352301 >. Acesso em: 15 out. 2019.

MORETTI, E. W.; ROBERTSON, K. M.; EL-MOALEM, H.; GAN, T. J. Intraoperative Colloid Administration Reduces Postoperative Nausea and Vomiting and Improves Postoperative Outcomes Compared with Crystalloid Administration. Anesthesia \& 
Analgesia. USA, v. 96, n. 2, p. 611-617, 2003. Disponível em: <https://insights.ovid.com/crossref?an=00000539-200302000-00056>. Acesso em: 15 out. 2019.

MYTHEN, M. G.; WEBB, A. R. Perioperative plasma volume expansion reduces the incidence of gut mucosal hypoperfusion during cardiac surgery. Archives of surgery. Chicago, v. 130, n. 4, p. 423-9, 1995. Disponível em: <http://www.ncbi.nlm.nih.gov/pubmed/7535996>. Acesso em: 16 out. 2019.

ØSTGAARD, G.; REED, R. K. Interstitial fluid accumulation does not influence oxygen uptake in the rabbit small intestine. Acta Anaesthesiologica Scandinavica. USA, v. 39, n. 2, p. 167-173, 1995. Wiley/Blackwell (10.1111). Disponível em: <http://doi.wiley.com/10.1111/j.1399-6576.1995.tb04037.x>. Acesso em: 15 out. 2019.

PEARSE, R.; DAWSON, D.; FAWCETT, J.; et al. Early goal-directed therapy after major surgery reduces complications and duration of hospital stay. A randomised, controlled trial [ISRCTN38797445]. Critical Care. New York, v. 9, n. 6, p. R687, 2005. Disponível em: <http://www.ncbi.nlm.nih.gov/pubmed/16356219>. Acesso em: 16 out. 2019.

PEREL, A.; HABICHER, M.; SANDER, M. Bench-to-bedside review: Functional hemodynamics during surgery - should it be used for all high-risk cases? Critical Care. New York, v. 17, n. 1, p. 203, 2013. BioMed Central. Disponível em: <http://ccforum.biomedcentral.com/articles/10.1186/cc11448>. Acesso em: 15 out. 2019.

POLDERMANS, D.; BOERSMA, E.; BAX, J. J.; et al. The Effect of Bisoprolol on Perioperative Mortality and Myocardial Infarction in High-Risk Patients Undergoing Vascular Surgery. New England Journal of Medicine. Canada, v. 341, n. 24, p. 17891794, 1999. Massachusetts Medical Society. Disponível em: <http://www.nejm.org/doi/abs/10.1056/NEJM199912093412402>. Acesso em: 15 out. 2019. 
RAOBAIKADY, R.; DINESH, S.; HACKING, M.; WIGMORE, T. Cardiopulmonary exercise testing as a screening test for perioperative management of major cancer surgery: a pilot study. Critical Care. New York, v. 11, n. Suppl 2, p. 250, 2007. BioMed Central. Disponível em: <http://ccforum.biomedcentral.com/articles/10.1186/cc5410>. Acesso em: 15 out. 2019.

RIELLA MC, RIELLA CV, PACHALY MA, RIELLA LV. Metabolismo da água. In: Riella $M C$, org. Princípios de nefrologia e distúrbios hidroeletrolíticos. $5^{\underline{a}}$ ed. Rio de Janeiro, Brasil: Guanabara Koogan; 2010. p. 105-38.

RUTTMANN, T. G.; JAMES, M. F.; VILJOEN, J. F. Haemodilution induces a hypercoagulable state. British Journal of Anaesthesia. United Kingdom, v. 76, n. 3, p. 412-414, 1996. Oxford University Press. Disponível em: <http://linkinghub.elsevier.com/retrieve/pii/S0007091217435483>. Acesso em: 16 out. 2019

SHOEMAKER, W. C.; APPEL, P. L.; KRAM, H. B.; WAXMAN, K.; LEE, T. S. Prospective trial of supranormal values of survivors as therapeutic goals in high-risk surgical patients. Chest. Glenview, v. 94, n. 6, p. 1176-86, 1988. Disponível em: <http://www.ncbi.nlm.nih.gov/pubmed/3191758>. Acesso em: 16 out. 2019.

SILVA, E. D.; PERRINO, A. C.; TERUYA, A.; et al. Brazilian Consensus on perioperative hemodynamic therapy goal guided in patients undergoing noncardiac surgery: fluid management strategy - produced by the São Paulo State Society of Anesthesiology (Sociedade de Anestesiologia do Estado de São Paulo - SAESP). Brazilian Journal of Anesthesiology (English Edition). São Paulo v. 66, n. 6, p. 557571, 2016. Sociedade Brasileira de Anestesiologia. Disponível em: $<$ https://linkinghub.elsevier.com/retrieve/pii/S0104001416301750>. Acesso em: 15 out. 2019.

SINCLAIR, S.; JAMES, S.; SINGER, M. Intraoperative intravascular volume optimisation and length of hospital stay after repair of proximal femoral fracture: randomised controlled trial. BMJ (Clinical research ed.). London, v. 315, n. 7113, p. 
909-12, 1997. Disponível em: <http://www.ncbi.nlm.nih.gov/pubmed/9361539>. Acesso em: 16 out. 2019.

SKYTTE LARSSON, J.; BRAGADOTTIR, G.; KRUMBHOLZ, V.; et al. Effects of acute plasma volume expansion on renal perfusion, filtration, and oxygenation after cardiac surgery: a randomized study on crystalloid vs colloid. British Journal of Anaesthesia. United Kingdom, v. 115, n. 5, p. 736-742, 2015. Oxford University Press. Disponível em: <https://linkinghub.elsevier.com/retrieve/pii/S0007091217310759>. Acesso em: 15 out. 2019.

SUBRAMANIAM, B.; SUBRAMANIAM, K.; PARK, K. W. Volume Replacement Strategies and Outcome. International Anesthesiology Clinics. USA, v. 48, n. 1, p. 115125, 2010. Disponível em: <https://insights.ovid.com/crossref?an=00004311201004810-00009>. Acesso em: 16 out. 2019.

TOCANTINS, L. M.; CARROLL, R. T.; HOLBURN, R. H. The Clot Accelerating Effect of Dilution on Blood and Plasma. Relation to the Mechanism of Coagulation of Normal and Hemophilic Blood. Blood. Washington, v. 6, n. 8, 1951. Disponível em: $<$ http://www.bloodjournal.org/content/6/8/720.short?sso-checked=true>. Acesso em: 15 out. 2019.

TOTE, S. P.; GROUNDS, R. M. Performing perioperative optimization of the high-risk surgical patient. British Journal of Anaesthesia. United Kingdom, v. 97, n. 1, p. 4-11, 2006. Oxford University Press. Disponível em: <http://linkinghub.elsevier.com/retrieve/pii/S0007091217351772>. Acesso em: 15 out. 2019.

\section{APÊNDICE - REFERÊNCIAS EM NOTA DE RODAPÉ}

2. Hamilton, 2011.

3. Holte, 2002.

4. Grocott, 2005. 
5. Brandstrup, 2012.

6. Lorentz, 2018.

7. Subramaniam, 2010.

8. Riella, 2010.

9. Lee, 1999.

10. Raobaikady, 2007.

11. Tote, 2006.

12. Skytte Larsson, 2015.

13. Moretti, 2003.

14. ØStgaard, 1995.

15. Gow, 1998.

16. Kellum, John.

17. Ruttmann, 1996.

18. Tocantins et al. 1951

19. Gurgel; Nascimento, 2011.

20. Hamilton, Cecconi, Rhodes, 2011.

21. Perel, Habicher, Sander, 2003.

22. Silva, Et al. 2016.

23. Bundgaard-Nielsen, 2007. 
24. Mythen, 1995.

25. Sinclair, Stephen. James, Sally. Singer, Merrill, 1997.

26. Pearse, Ruppert, 2005.

Enviado: Dezembro, 2019.

Aprovado: Janeiro, 2020. 